\title{
Oesterreichisches
}

\section{Botanisches Wochenblatt.}

Gemeinnütziges Organ

für

Botanik und Botaniker, Gärtner, Oekonomen, Forstmärnner, derzte, Apotheker und Techniker.

\section{Wien, 5. April 1855. v. Jahrgang. $\sqrt{1} 14$.}

Das Desterreichisehe botanische Wochenblatterscheint jeden Donnerstag. Ial pränumerirt auf dasselhe mit 4 fl. C. M. oder 2 Rthlr. 20 Ngr. jülerfich und zwar fur cxempl., die freidurcld die Post bezogen werden sollen, in as in der $\mathrm{s}$ eidel'nches Buchlandiung am Grabel in Wielt; ausserdem bei allen Buchlanbungen des In - und Auslandes. Inseratedie ganze Petitzeile $\tilde{z} \mathbf{k r}$. C. M.

Inhalt : Ueber Botrychium matricariaefolium und Polypodium Robertianum. Von Dr. M ild e. - Flora Lungau's. Von S t u r. - Vereine, Gesellschaften und Anstalten. - Mittheilungen. - Inserat.

\section{Ueber Rotrychiom matricariaefoltum A1. Braun. (R. rutacenn $\beta$ wahlemberg) und Polypodium Robertianum $\mathrm{H}$ of $\mathrm{m}$. (P. calcareum $\mathrm{S}$ in i $\mathrm{t}$ h.)}

Von Dr. Mild e.

Beim Studium der Literatur der deutschen Farrn fiel es mir auf, wie manche Arten in Bezug auf Beurtheilung von Seile der Botaniker ein recht eigenthümliches Schicksal gehabt haben. $\mathrm{Zu}$ den merkwürdigsten in dieser Hinsicht gehört das bekannte Botrychium matricariaefolium. W e b e $\mathrm{r}$ und Moh $\mathrm{r}$ betrachten es in ihrem botanischen Taschenbuche auf das Jahr 1807 mil B. rutaefolium Al. Braun (B. matricarioides Willd) als Varielät zu B. Lunaria. 0 . S $w$ a $\mathrm{r} \mathrm{z}$ vereinigt (1806) unter seinem rutaceum das rutaefolium und matricariaefolium, trennt jedoch noch Lunaria als besondere Art. Schkuhr war über die Deutung unserer Pflanze sich noch nicht klar und bildet auf 'Tafel 155 Fig. $b$ dieselbe $a b$, neben rataefolium (Fig. a derselben Tafel), welches er aus Schlesien, aus der Gegend von Oppeln erhalten, über welches er sich gleichfalls noch kein bestimmtes Urtheil gebildet hatte. Sommerse $t$ sah auf seiner Reise in das Stift Bergen deutlich, (Jahresberichte der k. schwed. Acad.), wie B. Lunaria und matricariaefolium ineinander übergingen; einige Exemplare waren völlige Lunaria, nur mit eingeschniltenen Blätichen, andere hatten kürzere Fruchtstiele und mehr eingeschnittene Blätter und wieder andere hatten das Blatt ebenso getheilt, aber kleiner. Strempel in seiner bekannten Dissertation stellt eine vollständige Uebergangsreihe von Lunaria matricariaefolium in instructiven Zeichnungen dar. Aehnlich spricht sich Burkhardt in der Flora 1849 


\section{6}

aus, der es bei Nislsy in der Oberlausitz beobachtete; er fand Lunaria und die fragliche Art durcheinanderwachsen, das erstere häufiger.

Der Ausgeber dieser Art in der ,Flora gall. et germ. exsicc. Cent. III. IV. $1840^{\circ}$ fand da, wo Lunaria wächst, auch diese Art; er sieht sie als eine Form von der ersteren an, deren Blätter sich mit Früchten bedecken wollen, was aber nur zum Theil gelingt, die Blätter nehmen die Gestalt der Trauben an, ein Theil des Parenchym verschwindet, und man findet hier und da Früchte auf den Blättern. Es ist daher eher eine Monstrositat. Alle schwedischen Botanilker betrachten es mit Fries als Varietät von Lunaria; ebenso Bernhardi, Kaulfuss, Hampe, Link, Roper, Doll, Rabenhorst. - Doch hat unsere Pflanze auch Vertheidiger, welche sie als gute Art hinstellen. M ouge ot betrachtet es in seimen Stirpes cryptog Vogeso-Rhenanae fasc. X. als eine von Lunaria wohl zu unterscheidende Art, welche 6 Wochen früher erscheine. - Dies ist jedoch nicht der Fall und kann nicht bestätigt werden. F. W. Schultz, der früher unsere Pflanze als Varietät betrachtele, stellt sie gegenwärlig als Art hin; ebenso Presl, Alex. Braun und Kli in mann.

Ich selbst batte nur zweimal Gelegenheit diese seltene Pflanze, welche der norddeutschen Ebene, in der sie in der neueren Zeit an vielen Orten beobachtet worden ist, (an einzelnen, wie bei Neudamm sogar zahlreich) eigenthümlich zu sein scheint, zu beobachten. Das erste Mal fand ich sie bei dem Bade Reinerz und zwar bei Reinerzkron, in einem einzigen ausgezeichneten Exemplare an einer ganz dürren, unfruchtbaren Stelle in Gesellschaft von Stereocaulon condensatum (1849), in der nächsten Umgebung war Lunaria sehr gemein, zu Hunderten und zeigte viele Abänderungen in Bezug auf seichtere und tiefere Einschnitte der Fiedern; doch gelang es mir nicht, einen vollständigen Uebergang zu Lunaria nachzuweisen. Im Jahre 1854 fand ich es auf dem höchsten Puncte des Ratschen (2465') auf Gneiss und Glimmerschiefer ganz in der Nähe des ersten Standortes (Kalkiger Sandstein,) in der allerengsten Gesellschaft mil Lunaria, welches hier an trockenen, grasigen Stellen zu Tausenden sich vorfand. Trotz des eifrigen Suchens konnte ich aber nur 3 Exemplare auftreiben, von denen nur eines vollsłändig war, während die beiden andern sehr verkïmmert, mit ganz schmalen, steilen Wedeln erschienen. Glücklicher war einer meiner Freunde, welcher diese Art von Frankenstein, gleichfalls in der Grafschaft Glaz, auf einem trockenen Hügel in Gesellschaft von Lunaria fand und zwar mit ziemlich deutlichen Uebergängen zu dieser Art. Ein Rhizom trug sogar ein Exemplar, welches ein evidentes Lanaria L. war, und ein zweites Exemplar, welches Anlage zu matricariaefolium zeigte. Wenige Ex́emplare wurden ausserdem bei Stohnsdorf auf dem Prudelberge, (am Riesengebirge) gefunden.

Ich betrachte unsere hicr behandelte Pflanze als eine durch den dürren Standort bedingte Form von Lunaria. Letzlere Pflanze erscheint an mehreren sehr fruchtbaren Stellen in Schlesien ungemein hoch und üppig; so z. B. in der kleinen Schneegrube und im Kessel 


\section{8}

des mährischen Gesenkes. Obgleich man hier Gelegenheit hat, Hunderie zu untersuchen, so wird man sich doch vergeblich nach bedentenden Abweichungen umsehen. Kommt jedoch Lunaria auf festen, dürren Boden, so bleibt es kleiner und zeigt auffallende Hinneigung zur Zertheilung der Fiedern, auffallend ist dann vorzüglich der dicke Stengel, dessen Verdickung vielleicht mit auf Kosten der Fiedersubstanz gescheht. Mit $B$. matricariaefolium muss nicht eine Form von Lunaria verwechselt werden, welche sehr selten vorzukommen scheint. Herr Dr. Kö rber fand sie auf dem keulichten Buchberg im Isergebirge (auf Basalt) in einem einzigen Exemplare, in Gesellschaft der Zwergform von $B$. Lanaria. Die Pflanze ist nur $1^{1 / 3}{ }^{\prime \prime}$ hoch, der Fruchtstengel den sterilen $1 / \mathbf{3}^{\prime \prime}$ überragend; letzterer im Umlange breit- deltaförmig, seine Spitze von einem aus schmaler, keilförmiger Basis sehr breiten, abgerundeten, nicht gespaltenen Fiederchen gebildet; die linke Seite des Wedels trägt 2 Fiedern, die rechte 3, von denen der unterste mit breiter abgerundeter Spitze, von 2 Paar normalen halbmondförmigen Fiederchen gebildet erscheint, so dass der Wedel am Grunde nicht einfach, sondern doppelt gefiedert ist. Eine räthselhafte Art bleibt nur jedoch das $B$. Kannenbergii Klins m a $n$. Ich hatte Gelegenheit 4 Exemplare desselben getrocknet zil sehen, und ich selbst besitze deren zwei. Sie ist bis jetzt in wenigen Exemplaren bei Memel und in einem bei Neudamm gefunden worden.

Die Beschaffenheit der Spitze des sterilen Wedels deutet offenbar auf eine Verwandtschaft mit B. Lunaria hin ; ja selbst die Gestalt der Fiedern ist bisweilen der von Lunaria ziemlich nahe kommend. Dagegen spricht wieder die tiefe Insertion des sterilen Wedels, sowie dessen langer Stiel, der bei der Lunaria stets fehlt, offenbar für eine Verwandtschaft mit rutaefolium Al. Braun. Sollte hier ein Bastard vorliegen?

Da ich $B$. Lunaria häufig zu beobachten Gelegenheit halle, so bin ich auf manche andere Abänderungen gestossen, die ich hiermit aufführe :

1) Es finden sich 2-4 fast gleichlange Fruchtrispen, aus einem und demselben Puncte entspringend.

2) Dem Hauptstengel sind etwas höher 1-2 andere Fruchtstengel angewachsen.

3) Ein zweiter Fruchtstengel entspringt aus der Rippe des sterilen Wedels.

4) Dasselbe Rhizom trägt $2-3$ vollständig ausgebildete Fflanzen.

5) Der Fruchtstengel fehlt ganz, nur der sterile ist vorhanden.

6) Die Fiedern des steriten Stengels sind mehr oder weniger mit Sporangien besetzt.

7) Der Stengel, welcher Fruchtrispe sein sollte, ist zum grössten Theil steriler Wedel geblieben, seine Fiedern spärlich mit Sporangien besetzt.

8) Der sterile Wedel ist bald nach seiner Ursprungstelle dichotom; beide Theile vollständig ausgebildet. 
Die zweite von uns zu betrachtende Pflanze ist das Polypodium Robertianum $\mathrm{H}$ of $\mathbf{f m}$. Schon $\mathrm{H}$ of $\mathrm{f} \mathrm{man} \mathbf{n}$ hebt in seinen Addenda zu Deutschlands Flora (1795) die drüsige Bekleidung als Kennzeichen hervor, und diese ist allerdings das einzige Kennzeichen neben einer gewissen Starrheit, wodurch es sich von Dryopteris unterscheidet; denn alle anderen Merkmale, die sich auf die Fiederung beziehen sollen, sind nicht stichhaltig; und so vereinigt es schon 1799 Bernh ardi in S ch ra ders Journal I. Bd. S. 306 mit Dryopteris als var. pinnulis angustioribus; auch $W$ ah l e $\mathrm{n} b \mathrm{erg}, \mathrm{Sp}$ en $\mathrm{n} \mathrm{r}$, Fries, Hampe, Sauter, Neilreich, F. W. Schultz sehen es nur als Varietát an, deren Auftreten, wie Einige angeben, durch den Standort bedingt sei. Die gewichtigsten Stimmen betrachten es dagegen als gute Art, zumal da diese Pflanze, wie ich dies auch in Schlesien, in Ustron bei Teschen, zu beobachten Gelegenheit hatte, in der allerengsten Gesellschaft von Dryopteris, und zwar nicht blos auf Kalk, sondern auch auf Grauwacke, vorkommt. 0. S w a rtz, Sprengel, Hoppe, Kaulfuss, Link, Tausch, Presl, Wallroth, Kunze, Döll, Wirtgen sehen sie als eine solche an. Ilier scheint mir die Entscheidung viel schwieriger, da es gar sehr fraglich ist, ob Drüsenbekleidung allein hinreichend ist, eine neue Art zu bilden; und doch sagt mir speziell eine geheime Stimme, unsere Pflanze als gute Art betrachten zu müssen. Hier könnte die Cultur entscheiden. Auf meine Veranlassung ist die Pflanze im hiesigen botanischen Garten ausgesäet worden, und ich werde seiner Zeit über das Resultat berichten Ueberhaupt sollte bei kritischen Arten vielmehr die Cultur angewendet werden, als dies bisher der Fall gewesen zu sein scheint, obgleich auf die Resultate derselben, nach meiner Meinung, nur nach einer Richtung hin ein entscheidendes Urtheil zu bauen ist. Geht z. B. aus den Sporen von Robertianum das Dryopteris hervor, dann ist ersteres allerdings als Varietät zu letzterem zu ziehen; wenn aus der Zucht jedoch wieder Robertianum entsteht, so folgt gewiss noch nicht daraus, dass dasselbe eine gute Art ist; denn evidente Varietäten pflanzen sich ja auch als solche fort. So wird es gewiss Keinem einfallen, wenn aus den Samen von Asplenium Adiantum niyrum var. serpentinum wieder dieselbe Pflanze hervorgeht, daraus auch zu schliessen, dass das A. Serpentini Ta usch eine gute Art und nicht vielmehr eine var. zu A. Adiantum nigrum ist, wie es schon die Beobachtung an ausgewachsenen Pflanzen lehrt.

Bresla u, im März 18o5.

\title{
Beitrag zur Kenntniss der Flora Lungau's.
}

\author{
Von D. S t ur.
}

(Fortsetzung.) (Hill)

Dianthus glacialis Hänke am Radstädter Tauern Dianthus sylvestris Wulf am Lug-Eck in der Mur auf Kl. Gl. 